\title{
Design and Implementation of Two-Degree-of-Freedom Nonlinear PID Controller for a Nonlinear Process
}

\author{
Rakesh $\mathrm{M} \mathrm{K}^{1} \quad$ Satheesh babu.R ${ }^{2}$ I. Thirunavukkarasu ${ }^{3 *}$
}

\begin{abstract}
A Two-Degree-of-Freedom (2-DOF) Nonlinear PID (NPID) controller is designed for a conical tank level process. The process is modeled using black box identification method as a First Order Process with Dead Time (FOPDT) model. Two nonlinear first order tracking differentiators are designed for the nonlinear PID controller. The closed loop control is implemented for a wide operating range of the process with the designed controller. The implementation and simulation is carried out using Mat-lab Simulink software. The online implementation of proposed controller shows that 2-DOF NPID is better than 1-DOF NPID.
\end{abstract}

Index Terms: Two-Degree-of-Freedom, First order process with dead time, Nonlinear PID, Nonlinear tracking differentiator

\section{Introduction}

A conical tank level process is a nonlinear process due to the conical shape of the tank. The change in area of the conical tank for equal rise in level is nonlinear. The nonlinear process is modeled as piecewise linear models at some operating points. This inherent nonlinear behavior of the processes makes control of such processes more complex. Presence of the nonlinearity in the plant often causes degraded performance of the overall process.

A nonlinear PID controller is developed by defining a nonlinear function which gives the controller settings depending upon the calculated error and some tuning parameters [1][2]. A nonlinear function may contain number of parameters and values of those should be decided by the designer and it varies from plant to plant.

When the process and the controller both exhibit nonlinear characters, a 2-DOF control scheme is considered to improve servo and regulatory problems [3]. In the 2-DOF control scheme the closed loop transfer functions for servo and regulatory can be adjusted independently [4][5][6]. In this work, the tuning of 2-DOF nonlinear PID controller is demonstrated to achieve stability and desired performance measures. This paper is organized such that first the design of the 2-DOF nonlinear PID control structure using the nonlinear tracking differentiators and secondly conical tank level process is described. Then the simulation study and online implementation of designed controller with actual process are shown.

\section{Nonlinear PID}

\section{2-DoF Nonlinear PID Control System}

A nonlinear function $f(e, \alpha, \delta)$ is used for the nonlinear PID as in [1] given by (1).

$f(e, \alpha, \delta)=\left\{\begin{array}{ll}\operatorname{sign}(e) .|e|^{\alpha}, & \text { when }|e|>\delta \\ \delta^{\alpha-1} e, & \text { when }|e| \leq \delta\end{array}\right\}$

The above is a highly nonlinear function with parameters $\alpha$ and $\delta$. The constant $\delta$ determines the linear range of the nonlinear function and $\alpha$ decides the shape the nonlinear function. The sign function is given in (2).

$$
\operatorname{sign}(A, \delta)=\left\{\begin{array}{ll}
\operatorname{sign}(A), & \text { when }|A|>0 \\
\frac{A}{\delta}, & \text { when }|A| \leq \delta, \delta>0
\end{array}\right\}
$$

Then, the control law can be written in parallel PID control form as in (3),

$u=K_{p} e f_{p}\left(e_{p}, \alpha_{p}, \delta_{p}\right)+K_{I} f_{I}\left(e_{I}, \alpha_{I}, \delta_{I}\right) \int e d t+K_{D} f_{D}\left(e_{D}, \alpha_{D}, \delta_{D}\right) \frac{d e}{d t}$

The NPID controller settings are tuned independently by individual tracking differentiators which makes it 2DOF control system. The 2-DOF NPID control structure is implemented as shown in Fig.1. 


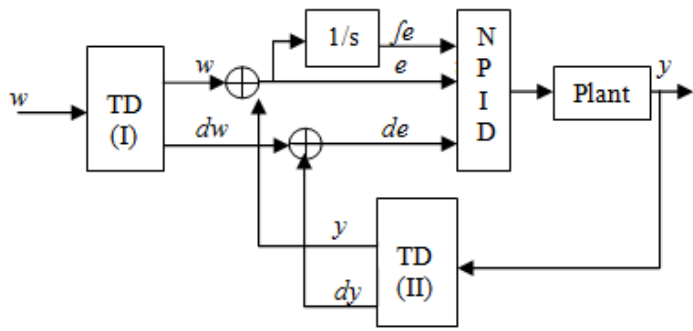

Fig.1. 2-DOF PID Control Structure

The proportional term is selected $\left(\delta_{\mathrm{P}} \leq 1\right.$ and $\left.0<\alpha_{\mathrm{P}}<1\right)$ as it should deliver large gain for small error and vice versa. For the integral action in order to avoid the integral saturation $1 \leq \alpha_{\mathrm{I}}<0$ is used. $\delta_{\mathrm{I}}$ is the parameter which decides the integral step which can be selected as small as $\delta_{\mathrm{I}} \leq 1$. The differential error is proportional to the proportional gain $\alpha_{\mathrm{D}} \leq 1$ and $\delta_{\mathrm{D}}$ can be selected appropriately [2].

\section{Nonlinear Tracking differentiator (NTD)}

A tracking differentiator gives the estimate of the derivative of the input and the tracked input. Use of a tracking differentiator makes the differential action more rapid and arrest the influence of noise signal up to some extent [8]. Two first order NTDs are used in the control structure, one for the set-point and the other in feedback path for the process variable.

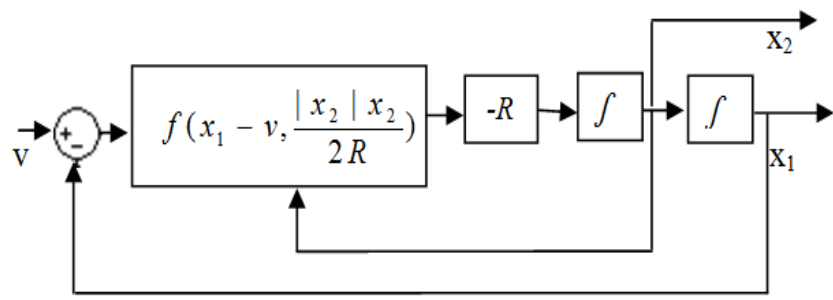

Fig.2. First order nonlinear tracking differentiator

Schematic representation of a first order NTD is given in Fig.2. A nonlinear function of input and two outputs by means of a feedback is combined to building NTD block and the same mathematically represented as follows in (4), where $\mathrm{R}$ is the tuning parameter.

$$
\left\{\begin{array}{l}
\frac{d x_{1}}{d t}=x_{2} \\
x_{2}=-R \operatorname{sign}\left(x_{1}-v(t)+\frac{\left|x_{2}\right| x_{2}}{2 R}\right)
\end{array}\right.
$$

\section{System Description}

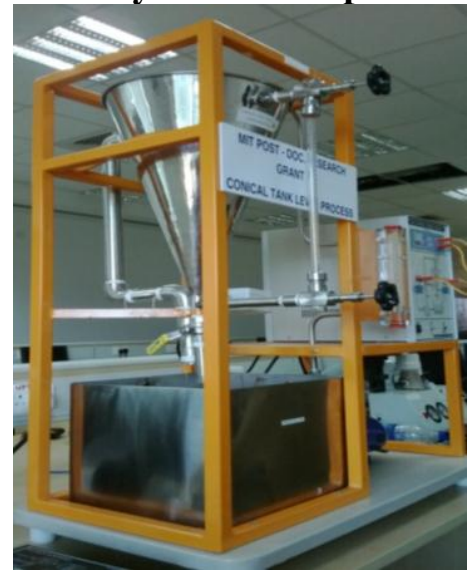

Fig.3. Conical Tank Level Process 
A Conical tank level process setup is shown in Fig.3. The conical tank is of $30 \mathrm{~cm}$ height. The final control element is a DC motor pump which can deliver max inflow of $1600 \mathrm{lph}$. A differential pressure transmitter is used to measure the level. The obtained process model is shown below in (5) which is modeled as $\mathrm{F}$

$P(s)=\frac{.925}{25.05 s+1} e^{-1.09 s}$

A conical tank level process is a nonlinear process due to the conical shape of the tank. The change in area of the conical tank for equal rise in level is nonlinear [9]. This inherent nonlinear behavior of the processes makes control of such processes more complex. Presence of the nonlinearity in the plant often causes degraded performance of the overall process and the process are modeled as piecewise linear models at some operating level points.

\section{Implementation and Results}

The tuned NPID parameter values are given in table I. The proportional gain $\left(\mathrm{K}_{\mathrm{P}}\right)$ with lesser values makes the process variable overshoot and higher value of the same cause steady state error. The large values of integral gain $\left(\mathrm{K}_{\mathrm{I}}\right)$ make the system asymptotically stable. $\mathrm{K}_{\mathrm{P}}$ and $\mathrm{K}_{\mathrm{I}}$ values are chosen suitably as 15 and 1.9. The derivative gain is chosen preferably very low to avoid the chattering effect. The parameters $\alpha_{\mathrm{P},} \alpha_{\mathrm{I}}, \alpha_{\mathrm{D}}, \delta_{\mathrm{P},} \delta_{\mathrm{I}}, \delta_{\mathrm{D}}, \delta_{1}$, and $\delta$ are defined in the range of zero and one.

Table I. Controller settings

\begin{tabular}{|c|c|c|c|}
\hline \multicolumn{2}{|c|}{1 -DOF NPID } & \multicolumn{2}{c|}{2 -DOF NPID } \\
\hline $\mathrm{K}_{\mathrm{P}}$ & 15 & $\mathrm{~K}_{\mathrm{P}}$ & 15 \\
\hline $\mathrm{K}_{\mathrm{I}}$ & 1.9 & $\mathrm{~K}_{\mathrm{I}}$ & 1.9 \\
\hline $\mathrm{K}_{\mathrm{D}}$ & .01 & $\mathrm{~K}_{\mathrm{D}}$ & .01 \\
\hline$\alpha_{\mathrm{P}}$ & 0.5 & $\alpha_{\mathrm{P}}$ & 0.5 \\
\hline$\alpha_{\mathrm{I}}$ & 0.5 & $\alpha_{\mathrm{I}}$ & 0.5 \\
\hline$\alpha_{\mathrm{D}}$ & 0.5 & $\alpha_{\mathrm{D}}$ & 0.5 \\
\hline$\delta_{\mathrm{P}}$ & 1 & $\delta_{\mathrm{P}}$ & 1 \\
\hline$\delta_{\mathrm{I}}$ & 1 & $\delta_{\mathrm{I}}$ & 1 \\
\hline$\delta_{\mathrm{D}}$ & 1 & $\delta_{\mathrm{D}}$ & 1 \\
\hline- & - & $\mathrm{R}_{1}$ & 10 \\
\hline- & - & $\mathrm{R}_{2}$ & 30 \\
\hline- & - & $\delta_{1}$ & .01 \\
\hline- & - & $\delta_{2}$ & .1 \\
\hline
\end{tabular}

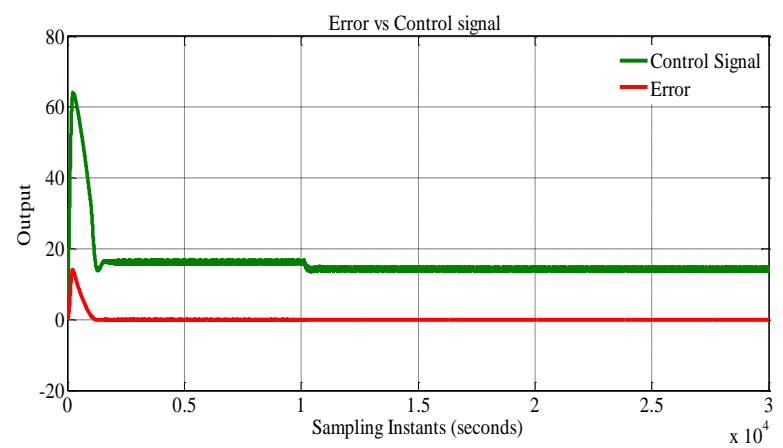

Fig.4. 2-DOF nonlinear PID controller output(simulation)

A nonlinear function may contain number of parameters and value of those should be decided by the designer and it varies from plant to plant. The simulated closed loop responses for a nominal value of $15 \mathrm{~cm}$ are shown in Fig. 4 and 5 respectively. A smooth nonlinear control action for the nonlinear level process with the 2DOF NPID makes the process variable to approach the set point immediately. $15 \%$ of nominal value is given as positive load and the loas is applied at 100s. 


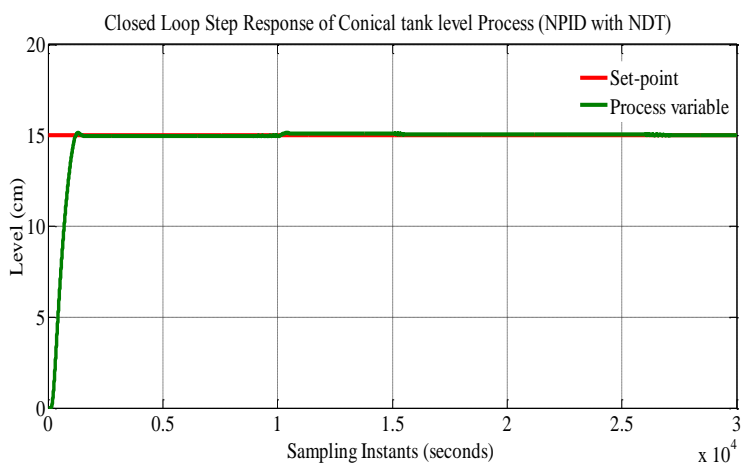

Fig.5. Simulated closed loop step response of process model with 2-DOF NPID Controller

The controller settings are tuned in simulation by reducing the integral absoulte error and the parameters are obtained as shown in table I. The nonlinear PID controller designed without an explicit model is implemented to control the whole operating range $(0-30 \mathrm{~cm})$ of conical tank.

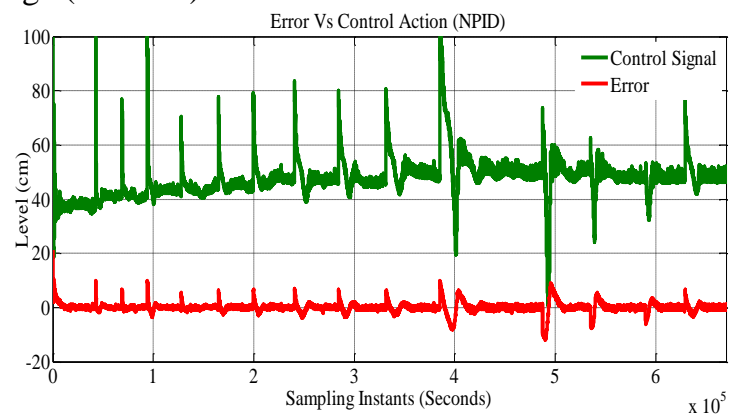

Fig.6. 1-DOF NPID controller output (real time)

Fig. 6 and 7 show that the control effort taken by the 1-DOF and 2-DOF nonlinear PID control schemes implemented on closed control of conical tank level process. The use of nonlinear NTD in 2-DOF control scheme reduces the effect of noise and chattering in system steady state response.

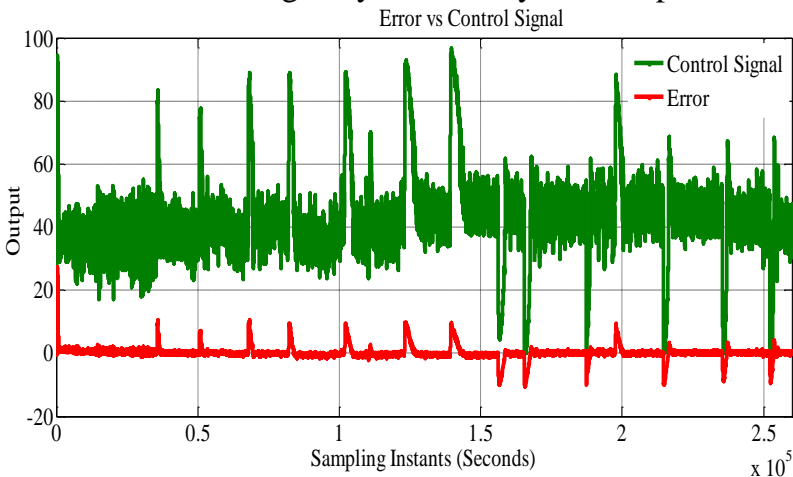

Fig.7. 2-DOF NPID controller output (real time)

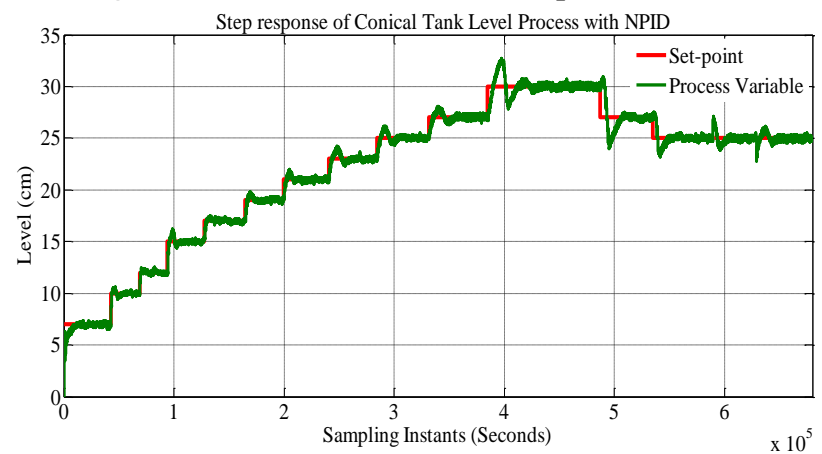

Fig.8. Closed loop response of conical tank level process with 1-DOF NPID controller (real time) 


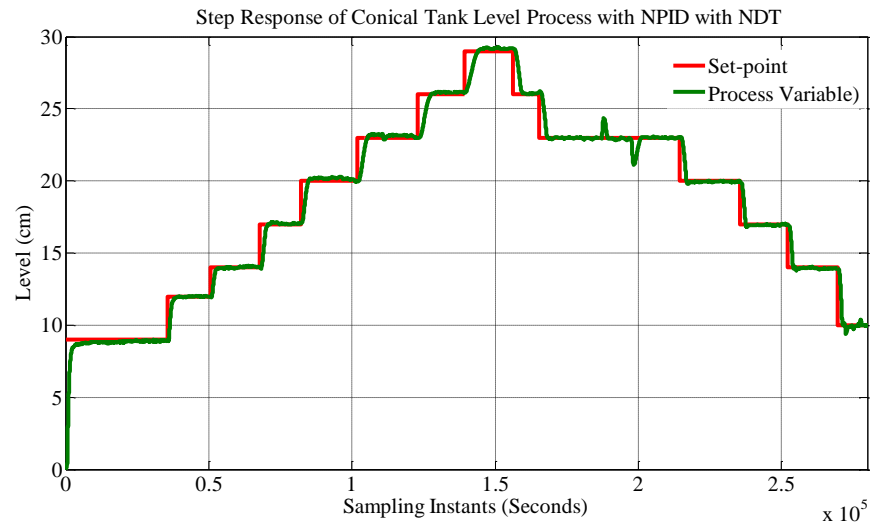

Fig.9. Closed loop response of conical tank level process with 2-DOF NPID controller (real time)

The real time plant response shown in Fig.8 with 1-DOF nonlinear PID controller corresponds to the control action in Fig.6 is. The response is having large overshoots especially at high operating points may end up in wind up effect while large set point are applied.

Table II. Controllers performances

\begin{tabular}{|c|c|c|c|c|c|c|c|}
\hline \multicolumn{4}{|c|}{ 1-DOF NPID } & \multicolumn{4}{|c|}{ 2-DOF NPID } \\
\hline Level $(\mathrm{cm})$ & $\begin{array}{l}\mathrm{T}_{\mathrm{r}} \\
(\mathrm{s})\end{array}$ & $\begin{array}{l}\mathrm{T}_{\mathrm{s}} \\
(\mathrm{s})\end{array}$ & $\begin{array}{c}\% \mathrm{M} \\
\mathrm{P}\end{array}$ & Level $(\mathrm{cm})$ & $\begin{array}{l}\mathrm{T}_{\mathrm{r}} \\
(\mathrm{s})\end{array}$ & $\begin{array}{l}\mathrm{T}_{\mathrm{s}} \\
(\mathrm{s})\end{array}$ & $\begin{array}{c}\% \\
\mathrm{MP} \\
\end{array}$ \\
\hline $0-7$ & 17 & 206 & 5 & $0-9$ & 11 & 70 & 0 \\
\hline $7-10$ & 10 & 170 & 20 & $9-12$ & 12 & 58 & 0 \\
\hline $10-12$ & 8 & 275 & 30 & $12-14$ & 20 & 121 & 7 \\
\hline $12-15$ & 18 & 172 & 40 & $14-17$ & 11 & 80 & 5 \\
\hline $15-17$ & 22 & 189 & 25 & $17-20$ & 12 & 86 & 9 \\
\hline $17-19$ & 21 & 134 & 40 & $20-23$ & 25 & 144 & 0 \\
\hline $19-21$ & 26 & 224 & 60 & $23-26$ & 32 & 79 & 10 \\
\hline $21-23$ & 28 & 344 & 65 & $26-29$ & 42 & 138 & 0 \\
\hline $23-25$ & 30 & 155 & 65 & $29-26$ & 29 & 43 & 0 \\
\hline $25-27$ & 32 & 336 & 67 & $26-23$ & 40 & 72 & 0 \\
\hline $27-30$ & 46 & 689 & 95 & $23-20$ & 38 & 70 & 0 \\
\hline $30-27$ & 44 & 379 & 102 & $20-17$ & 28 & 57 & 0 \\
\hline $27-25$ & 38 & 362 & 98 & $17-14$ & 24 & 42 & 0 \\
\hline $\begin{array}{l}\text { \% Load } \\
\text { applied }\end{array}$ & & $\begin{array}{l}\mathrm{T}_{\mathrm{s}} \\
(\mathrm{s})\end{array}$ & & $\begin{array}{l}\% \text { Load } \\
\text { applied }\end{array}$ & & $\begin{array}{l}\mathrm{T}_{\mathrm{s}} \\
(\mathrm{s})\end{array}$ & \\
\hline+10 & & 190 & & +10 & & 29 & \\
\hline-10 & & 207 & & -10 & & 43 & \\
\hline
\end{tabular}

The closed loop step response of the real time process with the 2-DOF nonlinear PID is shown in Fig.9. The plant tracks the set point efficiently with a satsfactory settling time. The over shoot and under shoot is arrested by the 2-DOF nonlinear control scheme. Both positive and negative load is given when the process is settled at $23 \mathrm{~cm}$. The load rejection is done effectively by the nonlinear PID as it can be observed in Fig.6. Camparison of designed controllers performances are provided in table II. Time domain performances are shown as $T_{r}$-rise time, $\mathrm{T}_{\mathrm{s}}$-settling time, MP-Overshoot. Load is applied as percentage of operating level.

\section{Conclusion}

The performance measures of 1-DOF and 2-DOF nonlinear PID controllers which are designed for the described conical tank level process are calculated and compared. It is found that the 2-DOF nonlinear control scheme is superior over 1-DOF by arresting the overshoot significantly and reducing the settling time and rise time. The modeling errors also can cause some complexity in control of processes especially nonlinear in nature The designed 2-DOF nonlinear PID control scheme is found as robust with the real time plant by offering excellent servo and regulatory responses. Apart from the difficulty in tuning of the control parameters the control scheme is found as improving the performance of 1-DOF nonlinear and conventional control schemes. 


\section{References}

[1]. Li Congying, et al, "Anti-windup Nonlinear PID Controller Design and its Application to Winged Missile Control System", Proceedings of the $27^{\text {th }}$ Chinese Control Conference,

[2]. Ma Shuhua, and Li Wei, "Nonlinear PID Control method with feed forward in smart car", Chinese Control and Decision Conference, pp.462-465, 2009

[3]. Araki, and Hidefumi Taguchi, "Tutorial Paper Two Degree of Freedom PID Controllers, "International Journal of Control, Automation, and Systems, Vol.1, No.4, pp.401-411, 2003

[4]. C.Shreesha, and R.D.Gudi, "Control relevant identification for two degrees of freedom control", Trans. IChemE, Vol 81,Part A.pp.679-688, 2003

[5]. M.Araki, and H.Taguchi, "Tutorial Paper, Two Degree of Freedom PID Controllers". International Journal of control, Automation and Systems Vol.1, No.4, pp.401-411, 2003

[6]. V.M.Alfaro et al, "Two-Degree-of-Freedom PI/PID tuning Approach for smooth Control on Cascade Control Systems", Proceedings IEEE Conference on Decision and Control, pp.5680-5685, 2008

[7]. Dapeng Tian et al, "New nonlinear tracking differential estimator:Thepry and practice", International journal of innovative computing, Information and control, Vol.1, No.4, pp.8257-8260, 2012.

[8]. Satheesh babu. R et al, "“Certain Discussions on 2DoF PID Control with Anti-Windup Schemes for the FOPDT”, CISCON'13, pp. 48,2013

[9]. Satheesh babu. R et al, “Tuning of 2Degree-of-Freedom PID Controller for a Nonlinear Process”, TIMA-2013, pp. 117-120, 2013

\footnotetext{
I. PG Student, Department of I\&C, MIT, Manipal, India. Email: - rakeshmk@outlook.com

2. JRF, Department of I\&C, MIT, Manipal, India.

3*. Associate Professor, Department of I\&C, MIT, Manipal, India.
} 